\title{
Editorials
}

\section{The vegetative state - clinical diagnosis}

Rare conditions occasionally have a major impact on medicine and society. The vegetative state is one such disorder - not only in the controversies of how to treat but also whether to treat. The case of Tony Bland, a young man becoming vegetative due to severe anoxic brain damage when he was crushed in the Hillsborough football disaster, hit the headlines and has been the basis for much debate about withdrawal of nutrition and hydration, the right to die and euthanasia. The very high cost of caring for vegetative patients over several decades has important implications in legal cases and in the use of resources. There is still, however, a considerable misunderstanding about the condition.

\section{Terminology}

In spite of attempts to have clear terminology, Giacino and Zasler ${ }^{1}$ have pointed out that there is still "a relative lack of understanding of the existing nomenclature and a tendency toward inappropriate diagnoses and subsequently incorrect conclusions regarding neurological and functional prognoses and necessary treatment".

The term 'persistent vegetative state' (PVS) was coined by Jennett and Plum ${ }^{2}$ to describe a specific syndrome of reflex reactions without any meaningful response to the environment but in patients who have a sleep-awake pattern. They introduced the term because they were generally unhappy about the other terms used at the time. These terms either use categories which were not true, eg, prolonged $c o m a^{3}{ }^{4}$ or coma vigil ${ }^{5}$ (when the patients, by definition, were not in coma), or were of specific syndromes, eg, decerebrate dementia, ${ }^{6}$ parasomnia, ${ }^{7}$ or akinetic mutism. ${ }^{8}$ The term apallic syndrome, ${ }^{9}$ still used in Germany, implies lack of the cortex which may not be the case, especially in traumatic injuries.

There is nevertheless a dislike, especially amongst relatives of brain-damaged people, of the term 'vegetative' mainly because of its association with the word 'vegetable'. Sadly, the medical profession is not immune to talking about patients as being 'permanent vegetables'.

There has been an attempt in Israel by Groswasser and Sazbon ${ }^{10}$ to use the term 'post-comatose unawareness' but this has not caught on in the rest of the world, probably because the term 'vegetative state' is so ingrained in medical and legal terminology that it is difficult to accept a new term which is more wieldy. They suggested that their term had the advantages that it described the clinical picture of the patient who is no longer in coma but is not showing any evidence of awareness, whilst not using arbitrary and retrospective criteria of time nor implying one single outcome

In addition some concern has been expressed about attaching the terms persistent and permanent in the terminology of the vegetative state. The Multi-Society Task Force on PVS $^{1112}$ point out that confusion has arisen over the exact meaning of the word persistent. They expressed the view that 'persistent vegetative state' was a diagnosis, while 'permanent vegetative state' was a prognosis. The Multi-Society Task Force and others have placed arbitrary time periods on these terms with the term persistent usually describing those patients vegetative for more than one, three or 12 months according to aetiology; and the term permanent for those vegetative from a traumatic cause for more than one year.

Several international working groups ${ }^{13}$ have recommended the terms persistent and permanent be dropped, whilst the Royal College of Physicians ${ }^{14}$ has suggested that the word persistent be replaced by the term continuing.

\section{Clinical features}

The clinical description by Jennett and Plum $^{2}$ has been further developed by the by the Multi-Society Task Force on PVS ${ }^{112}$ and has been approved by the American Academy of Neurology, Child Neurology Society, American Neurological Association, American Association of Neurological Surgeons, and the American Academy of Paediatrics.

The generally accepted features of the vegetative state based on these various reports are:

- After a period of coma the patient opens his/her eyes, at first to pain and then to less arousing stimuli. This is then followed by periods with the eyes open. This intermittent wakefulness, manifest by sleep-wake cycles, is the point of evolution from coma. Some authorities do not like to talk in terms of 'sleep-awake' since this implies a higher cortical function. They prefer to use the simple terms of eye opening and closing. It is very difficult, however, to persuade caring staff and relatives not to talk in terms of asleep and awake.

- The patient may blink to menace but appear not to be attentive. It is of note that other authorities ${ }^{14}$ have regarded a blink to threat as evidence of cortical connection and therefore indicating that the patient is not vegetative. This is a very questionable approach since the concept of the vegetative state is the demonstration of awareness, not whether there are some cortical connections. The Multi-Society Task Force ${ }^{11}$ urges caution in making the diagnosis of the vegetative state if there is blinking to threat but does not go as far as to claim that, if present, it indicates that the patient is no longer vegetative.

- There may be roving eye movements and the patient's eyes may seem to briefly follow moving objects. The movement is usually inconsistent and never sustained. The main early sign that the patient is emerging from the vegetative state is that he begins to focus on and/or tracks a moving object or person. The difficulty here is that Andrews et al ${ }^{15}$ found that $60 \%$ of patients who were misdiagnosed as being vegetative were blind or had severe visual impairments which would make focussing an impossibility.

- The predominant feature is that all responses are reflex in nature. In the vegetative state these present as:

(a) No evidence of sustained, reproducible, purposeful, or voluntary behavioural responses to visual, auditory, tactile, or noxious stimuli. The Aspen Consensus Group have emphasised that if any of these parameters (sustained, reproducible, purposeful or voluntary responses) are present then the diagnosis of the vegetative state should be made with caution. If any of these responses occur but are not consistent then a safer diagnosis is that of the minimal conscious state. 
(b) Reflex posturing. This is common with any severe brain damage, even when there is awareness.

(c) Flexor withdrawal occurs but usually after a delay and never takes the form of a brisk response. The response is slow and dystonic

(d) A non-volitional grasp reflex may be present. This can cause considerable concern to relatives who feel that the patient recognises them when they hold his hand.

(e) Fragments of co-ordinated movement, such as scratching or even moving hands towards a noxious stimulus may occur.

(f) Reflex postural alterations of the limbs may be provoked by neck movements.

(g) Chewing movements or grinding of teeth, sometimes accompanied by constant movement of the tongue. These again cause concern to relatives who may feel that the patient is indicating that he is thirsty or hungry.

(h) Liquid and food placed in the mouth may be swallowed. A few vegetative patients can take all their nourishment orally. In the vast majority of patients, however, there is gross disturbance of the swallowing mechanism.

(i) Grunting and groaning may be provoked by noxious stimuli but no speech occurs. These sounds are often interpreted by relatives as indicating an attempt to communicate. This can cause disagreement between family and clinicians when some relatives claim to be able to 'understand' the words spoken when others only hear sounds.

(j) Sufficiently preserved hypothalamic and brainstem automatic functions to permit survival with medical and nursing care. Basically this means that the patient does not require ventilation and breathes spontaneously.

- No evidence of awareness of self or environment and an inability to react with others or have any meaningful response to the spoken word. This begs the question "meaningful to whom?". Giacino and Zasler ${ }^{1}$ have pointed out that there is no method yet available to clinically assess 'internal awareness' in a patient who is otherwise unable to express awareness relative to external environmental stimuli. The concept that we are only able to infer the presence or absence of conscious experience has also been pointed out by Bernat ${ }^{16}$ and the Multi-Society Task Force. ${ }^{11}$ The International Working Party on the Vegetative State ${ }^{13}$ discussed this point in detail and criticised the use of the term 'meaningful response' on the grounds that it requires a considerable amount of subjective interpretation on the part of the observer and that what was meaningful for the patient may not be considered meaningful by those treating the patient. Similarly the term 'purposeful response' was criticised because of the subjective interpretation and that a withdrawal reflex could be considered as purposeful in that it removes the limb, for instance, from danger.

The Royal College of Physicians ${ }^{14}$ has more recently defined a code of practice following the recommendations of the House of Lords Select Committee on Medical Ethics. Their criteria for the vegetative state were very similar to the above definitions except that they added "There will not be nystagmus in response to ice water caloric testing, the patient will not have visual fixation, be able to track moving objects with the eyes or show a 'menace' response". Whilst tracking moving objects is generally regarded as evidence of some form of awareness, the other tests indicate a neuronal connection to the cortex but, as far as I am concerned, do not imply that the patient is aware.

It is important to recognise that the vegetative state is a syndrome of clinical features and not a pathological, anatomical or disease process diagnosis. The emphasis is on behavioural responses demonstrating awareness. It is important to differentiate the vegetative state from:

Coma: In this state there are no verbal response, the patient does not obey commands, responses are absent or at a primitive reflex level and the patient does not open his eyes either spontaneously or to any stimulus. ${ }^{17}$

Brain death: In this there are absent brainstem reflexes, the patient is apnoeic, presents in coma, shows no abnormal posturing (either decorticate or decerebrate) and there is no epileptic jerking. These patients need artificial ventilation to survive since they are unable to breathe spontaneously. ${ }^{18}$

Locked-in syndrome: In this state the patient is totally paralysed and can usually only respond by eye movement and even this is in a limited direction. These patients often have full mental function. It is important to recognise this group of patients since so much can be achieved with modern technology to enable them to take control of their lives.

There is another group of profoundly head injured people who are not true locked-in patients but have a combination of profound physical disability combined with some cognitive impairment. This makes diagnosing the level of awareness particularly difficult and requires considerable amount of skill and experience to obtain optimal evaluation.

\section{Post-vegetative state}

There is difficulty in finding a suitable term for those patients who no longer fit the criteria for the vegetative state but have not returned to levels of normal communication. There is a state where the patient has progressed from the vegetative state and is making definite responses to the internal (eg, pain and bowel or bladder discomfort) and/or external environment which could be interpreted as being an expression of awareness.

There is some concern about the commonly used term 'low awareness state' since it is argued that even fully mentally alert people may, albeit temporarily, be 'unaware' of their surrounding when absorbed in other activities. Giacino $e t a l^{19}$ have suggested the term 'minimally responsive state'. Again this has limitations in that all vegetative state patients have minimal responses, albeit at a subcortical reflex level.

Recently, the influential Aspen Consensus Group on Brain Injury in the United States has coined the term 'minimal conscious state' (yet to be published). This term has provisionally been agreed by a European working group on the vegetative state. The features are movements and responses which cannot be explained by reflex patterns and but which are not occurring consistently enough to enable reliable use of these responses for communication purposes. This state is the transition stage between being in the vegetative state and the ability to communicate consistently, although some patients may remain at this level permanently.

\section{Difficulty in diagnosis}

The International Working Party on Vegetative State ${ }^{13}$ recognised that deciding the cognitive awareness of this type of patient, especially when at a very low level, was an educated guess since there are, as yet, no tests which can confirm whether the patient has any 'inner awareness'. They pointed out that the assessments in general use are based on a series of behavioural patterns. The clinician is, therefore, dependent on overt responses which depend on:

- the physical ability of the patient to respond

- the desire or willingness (if the patient is aware) of the patient to respond 
- the abilities of the observer to make rapport with the patient

- the ability to observe accurately

- the time available for observation and assessment

- the lack of available and reliable assessment tools.

It is essential to understand that the diagnosis is based on the presentation of clinical features since it is basically a behavioural model. There are three prime features of the vegetative state. The first is that the patient has sleep-awake patterns; this differentiates the condition from coma. This is a feature which can be easily detected by the lay person. There is the theoretical possibility that a patient with bilateral third nerve palsy could be aware but have no eye opening. I have only come across one case (in America) where this has been shown to have happened. The second feature is that all responses can be identified as reflex patterns. This requires considerable neurological knowledge of reflex patterns. The third element, the other side of the coin to reflex patterns, is that the patient makes no meaningful responses and has no awareness. This is much more difficult since the only way anyone can demonstrate their awareness is through a motor function, ie, speech, facial expression, or physical gesture. In the presence of severe spasticity, muscle inactivity and dysphasia it is extremely difficult for even an aware person to demonstrate their awareness.

This difficulty has been seen in several studies. Childs et $a l^{20}$ report that $37 \%$ of patients admitted more than one month post injury with a diagnosis of coma or persistent vegetative state had some level of awareness. In a group of longer term patients in a nursing home, Tresch $e t a l^{1}$ found that $18 \%$ of those diagnosed as being in the persistent vegetative state were aware of themselves or their environment.

The study by Andrews et $a l^{15}$ highlights some of the major problems in making a diagnosis of the vegetative state. They reviewed the records of 40 consecutive patients admitted to their specialist profound brain injury unit at least 6 months following their brain injury (a period after which spontaneous recovery is generally regards as limited) with a referral diagnosis of vegetative state. They found that whilst $25 \%$ remained vegetative, $33 \%$ emerged during the rehabilitation programme, and $43 \%$ had been misdiagnosed $(41 \%$ of these for more than a year including three for more than 5 years). The level of cognitive functioning present in this misdiagnosed group at the time of discharge was considerable: $60 \%$ were orientated in time, place and person, $75 \%$ were able to recall a name after 15 minutes delay, $69 \%$ were able to carry out simple mental arithmetic, $75 \%$ were able to generate words to communicate their needs and $86 \%$ were able to make choices about their daily social activities.

The predominant features which seem to have lead to misdiagnosis were that all the patients were profoundly physically disabled and $60 \%$ were blind or severely visually impaired. Since clinicians use visual tracking as an important sign that the patient is emerging from the vegetative state, and this sign was usually absent because of the damage to the visual tracts, the patients were mistakenly assumed to be vegetative.

There are several prerequisites for the accurate assessment of the person thought to be in the vegetative state:

- The patient should be healthy. Even simple conditions such as constipation, chronic urinary tract infection (usually associated with long term catheterisation) or chest infections can prevent optimal responses from being obtained.

- The patient should be in a good nutritional state. Until recently as many as $80 \%$ patients admitted to our specialist unit were suffering from undernutrition. The earlier use of gastrostomy feeding has altered this pattern but still about $30 \%$ of patients admitted have a low Body Mass Index, emphasising the difficulty in managing people with complex medical and physical disabilities.

- As many sedating drugs as possible should be withdrawn, or at least decreased to the lowest effective dose; this includes antispasticity and anti-epileptic drugs. In the case of anti-epileptic drugs which are still required to control fitting, drugs with the least sedative effect should be used.

- Complications and consequences of neurological imbalance should be prevented; this includes high muscle tone and contractures by the provision of special seating, good bed and sitting posture to control abnormal muscle tone. These complications in the long term increase the amount of nursing care required which, since the patient may live for many years, increases the cost of care considerably.

- Controlled posture is important. Most doctors have been trained to examine patients on the bed. Experience suggests that patients are more likely to be alert when sitting up (presumably due to greater stimulation of the reticular activating system). A well supporting seating system is essential to reduce sufficient muscle tone to allow movement of limbs which can be used for communication purposes, eg, to press a touch-sensitive switch.

- Providing a controlled environment of sensory regulation to avoid sensory overload of the severely damaged brain. Since it is likely that profoundly brain-damaged patients have a problems with selective attention, sensory input should be simple and interspersed with periods of rest. It is, therefore, logical to assess for cognitive responses after a period of rest rather than after a period of activity, such as being washed and dressed, or after a period of physiotherapy. This requires staff and family to understand the importance of avoiding overstimulation prior to the assessment.

- Assessments should be short (to avoid tiring the patient), repeated (to identify windows of opportunity) and carried out over a period of time (to accommodate the learning process of both the patient and the assessor). A short, one-off, assessment of the patient who is lying in bed is likely to result in a missed diagnosis, even by very experience clinicians.

- The ability to generate a behavioural response fluctuates from day to day and hour to hour, and even minute to minute, depending on fatigue factors, general health of the patient and the underlying neurological condition.

- Observation needs to take into account delayed responses. Assimilation of even basic information is often slow and therefore response time may be delayed. Because of this, information provided at any one time should be simple, consistent, repeated after a period of rest and allow for a delayed response.

- Communication requires skilled techniques and a sensitivity for the method by which the patient wants to communicate.

- Families and other carers have a very important role in identifying the best responses and the optimal conditions for assessment. Whilst there are some relatives who interpret reflex responses as being meaningful, there is no doubt that members of the family are often more sensitive to early changes than even experienced clinical staff.

\section{The value of neurophysiological investigations}

I have not discussed the use of neurophysiological assessment. Diagnosis is by definition a clinical one since it 
depends on behavioural responses which, by their very nature, require a subjective element for assessment. There is, therefore, a great need for more objective approaches. The International Working Party on the Vegetative State ${ }^{13}$ came to the conclusion that, at present, electrophysiological and radiological examinations could be supportive of the clinical diagnosis but could not per se be diagnostic or prognostic. The more dynamic neurophysiological assessments such as functional magnetic resonance imaging and brain mapping offer the best opportunity so far but have yet to be demonstrated to be accurate enough for day-to-day use.

\section{Summary and conclusion}

The vegetative state is a group of clinical features of profound brain damage which attempt to demonstrate that

1 Giacino JT, Zasler ND Outcome after severe traumatic brain injury: coma, vegetative state and the minimally responsive state. F Head Trauma Rehabil 1995;10:40-56.

2 Jennett B, Plum F. Persistent vegetative state after brain damage: a syndrome looking for a name. Lancet 1972;i:734-7.

3 Nakamura N, Hirakawa K, Zimbo M, Sano K, Mukai N. Prolonged posttraumatic coma. I Clinical aspects. Brain Nerve (Tokyo) 1965;17:98998.

4 Crompton MR, Teare RD, Bowen DAL. Prolonged coma after head injury. Lancet 1966;i:938-40.

5 Gerstenbrand F. Das traumatische apallisches Syndrome. [The traumatic apallic syndrome.] Berlin: Springer-Verlag, 1967.

6 Roberts AH. Sequelæ of closed head injury. Proc R Soc Med 1976;69:137-

7 Jefferson G. The nature of concussion. BM7 1944;1:1-5

7 Jefferson G. The nature of concussion. BMF $1944 ; 1: 1-5$. with an epidermoid cyst of the third ventricle. Brain 1941;64:273-90. With an epidermoid cyst of the third ventricle. Brain 1941;64:273-90.

10 Kretschmer E. Das apallisches Syndrome. Z Neurol 1940;169:576-9. matic unawareness. Part 2: Functional outcome of 72 patients recovering consciousness. F Neurosurg 1990;72:81-4.

11 The Multi-Society Task Force on PVS. Medical aspects of the persistent vegetative state (first of two parts). N Engl F Med 1994;330:1499-508.

12 The Multi-Society Task Force on PVS. Medical aspects of the persistent vegetative state (second of two parts). N Engl F Med 1994;330:1572-9. the patient is awake but unaware of internal or external environments. The diagnosis depends on an assessment of behavioural responses. Because of the complexity of the clinical features, it takes considerable experience to have confidence in the diagnosis. It is certainly not a diagnosis which can be made on a one-off assessment at the bedside without a considerable amount of supportive evidence from a multidisciplinary team experienced in the management of severe brain damage. So far, neurophysiological investigations can at best be supportive, rather than diagnostic.

KEITH ANDREWS

Director of Medical \& Research Services, Roval Hospital for Neuro-disability, West Hill, Putney, London, UK

Accepted 15 January 1999

Keywords: vegetative state

13 Anon. International Working party on the Vegetative State. London: Royal Hospital for Neuro-disability, 1995.

14 Working Group of the Royal College of Physicians. The permanent vegetative state. $\mathcal{F} R$ Coll Physicians London 1996;30:119-21.

15 Andrews K, Murphy L, Munday R, Littlewood C. Misdiagnosis of the vegetative state: retrospective study in a rehabilitation unit. BMF 1996;313:13-

16 Bernat JL. The boundaries of the persistent vegetative state. $\mathcal{F}$ Clin Ethics 1992;3:176-80.

17 Jennett B, Teasdale G. Aspects of coma after severe head injury. Lancet $1977 ; \mathbf{i}: 878-81$.

18 Pallis C, Harley DH. Diagnosis of brainstem death-2. In: Pallis C, Harley $\mathrm{DH}$, eds, $A B C$ of brainstem death, 2nd edn. London: BMA Publishing Group, 1996; pp 17-20.

19 Giacino JT, Zasler ND, Whyte J, Kate DI, Glenn M, Andary M. Recommendations for the use of uniform nomenclature pertinent to patients with severe alterations in consciousness. Arch Phys Med Rehabil 1995;76:203-7.

20 Childs NL, Mercer WN, Childs HW. Accuracy of diagnosis of persistent vegetative state. Neurology 1993;43:1465-7.

21 Tresch DD, Farrol HS, Duthie EH, Goldstein MD, Lane PS. Clinical characteristics of patients in the persistent vegetative state. Arch Intern Med 1991;151:930-2. 